\title{
Bunching near transition in the BNL AGS
}

\author{
C. Ankenbrandt, K-Y. Ng, J. Norem,* M. Popovic, and Z. Qian \\ Fermi National Accelerator Laboratory, Batavia, Illinois 60510
}

\author{
L. A. Ahrens, M. Brennan, V. Mane, T. Roser, D. Trbojevic, and W. van Asselt \\ Brookhaven National Laboratory, Upton, New York 11973 \\ (Received 28 January 1998; published 13 July 1998)
}

\begin{abstract}
The proton driver for the muon collider must produce short pulses of protons in order to facilitate muon cooling and operation with polarized beams. In order to test methods of producing these bunches, we have operated the alternating-gradient synchrotron near transition and studied procedures which involved moving the transition energy $\gamma_{t}$ to the beam energy. We were able to produce stable bunches of $3-5 \times 10^{12}$ protons with rms widths of $\sigma=2.0-2.7 \mathrm{~ns}$ for longitudinal bunch areas of $1.5 \mathrm{eV} \mathrm{s}$, in addition to making measurements of the lowest two orders of the momentum compaction factor. [S1098-4402(98)00008-1]
\end{abstract}

PACS numbers: 29.20.-c, 29.27.Ac, 29.27.Bd, 29.27.Fh

\section{INTRODUCTION}

Muon collider designs [1,2] require that the pions from which muons are produced are generated in a short bunch, both to facilitate subsequent cooling and to improve the separation between polarization states. Calculations done for the feasibility study imply that the required initial $\mathrm{rms}$ bunch length from the proton driver [3] is $\sigma \sim 1-2$ ns, more than a factor of 3 shorter than the natural bunch length in the Brookhaven National Laboratory Alternating-Gradient Synchrotron (BNL AGS) [4]. Several methods of producing short bunches were considered in that study, including bunch expansion followed by rf bunch rotation [5], rebunching at a higher frequency [6], operation near transition where the bunch length is naturally short [2], or the use of specialized linacs, beam lines, and bunching rings [7].

Since the proton driver contemplated for the muon collider could have its extraction energy near the natural transition energy [8], and would probably use a flexible momentum compaction (FMC) [9] lattice with easily adjustable transition energy, we have looked at options which could make use of transition in bunching. This paper summarizes simulations and experiments on the AGS near transition.

\section{BUNCHING NEAR TRANSITION}

Although a number of methods of bunching are possible, the primary methods which have been considered for the proton driver of the muon collider are (i) adiabatic spreading of the bunch followed by bunch rotation, (ii) rebunching the beam at a higher frequency, and (iii) energy

*Permanent address: HEP Div., Argonne National Laboratory, Argonne, IL 60439. spreading near transition, followed by a partial rf bunch rotation with the transition energy moved far enough from the beam energy to ensure that the bunch rotation was fast. Combinations of these methods, for example, bunch rotations in two successive synchrotrons, have also been considered. Of these methods, (i) and (ii) are comparatively common and well understood, but (iii) involves operation near transition, which is comparatively uncommon. A recent development is the invention of the FMC lattice, which permits control of the transition energy over a very wide range, with minimal interactions with the rest of the machine. Calculations have shown that the transition energy can be changed by factors of 2 by a small adjustment of two quadrupoles without changing the tunes of the machine [10].

Bunching near transition is complicated by the nonlinearity in phase shear which introduces nonlinear motion in synchrotron space, as well as the small value of $|\eta|$ which reduces the synchrotron frequency, making these nonlinearities more prominent. Following reference [11], the expression for the circumference

$$
C(\delta)=C_{0}\left[1+\alpha_{0} \delta\left(1+\alpha_{1} \delta+\alpha_{2} \delta^{2}\right)+\ldots\right],
$$

where $\Delta p / p=\delta, C_{0}$ is the median circumference $(\delta=$ $0)$, and the $\alpha$ 's are various orders of the momentum compaction. One also defines the slip factor as a function of the revolution time $T$

$$
\eta \equiv \frac{\Delta T}{T} / \delta
$$

giving the transition gamma

$$
\gamma_{t}(\delta)=\gamma_{t 0}\left[1-\left(\alpha_{1}+0.5-\alpha_{0} / 2\right) \delta+O\left(\delta^{2}\right)\right]
$$

and

$\eta(\delta) \sim 1 / \gamma_{t}^{2}-1 / \gamma^{2}+\left(1 / \gamma_{t}^{2}\right)\left(\alpha_{1}+1.5 \beta\right) \delta+O\left(\delta^{2}\right)$. 
The first order momentum compaction is therefore

$$
\alpha_{1}=-\frac{1}{\gamma_{t 0}} \frac{d \gamma_{t}}{d \delta}-1 / 2+\alpha_{0} / 2
$$

In the equations above, $\gamma_{t}$ is the transition energy, $\alpha_{0}=$ $1 / \gamma_{t}^{2}, \gamma=E / m_{p}, E$ is the total energy, $\beta=v / c=$ 0.993 for this test, $m_{p}$ is the proton mass, and the subscript 0 refers to the nominal beam energy. Far from transition, the difference between the first two terms of Eq. (4) dominates and $\eta(\delta)$ is essentially a constant; however, close to transition, the first two terms cancel each other and $\eta(\delta) \propto \delta$. Since the shear or slip in synchrotron space $(\delta, \phi)$ is equal to $\eta \delta$, if both $\eta$ and $\delta$ depend linearly on momentum, the product is quadratic. Thus the general bunch deformation is a shear in the $\phi$ direction, which depends quadratically on $\delta$ within the bunch. If $\eta$ can be made constant over the range of $\delta$ in the bunch, by requiring $\alpha_{1}=-1.5$, the quadratic deformation will be removed. The $O\left(\delta^{2}\right)$ term is assumed to be small, since it is difficult to design precise measurements even of the linear term.

A plot of the $\gamma_{t}$ against $\delta$ as a function of sextupole and octupole settings using methodical accelerator design (MAD) [12], Fig. 1, shows that it is, in principle, possible to match $d \gamma_{t} / d \delta$ of the machine to the beam over a large range of bunch momenta, although the capability of MAD to compute the higher orders of momentum compaction may be questionable, due to closed orbit distortions in the real machine. With sextupoles off, $\eta$ decreases with $\delta$; however, the slope $d \gamma_{t} / d \delta$ and the quadratic component increase with horizontal sextupole current $I_{\mathrm{Hs}}$. The quadratic component can be canceled with octupoles. Predictions of the effect of sextupole and octupole correctors on the momentum dependence

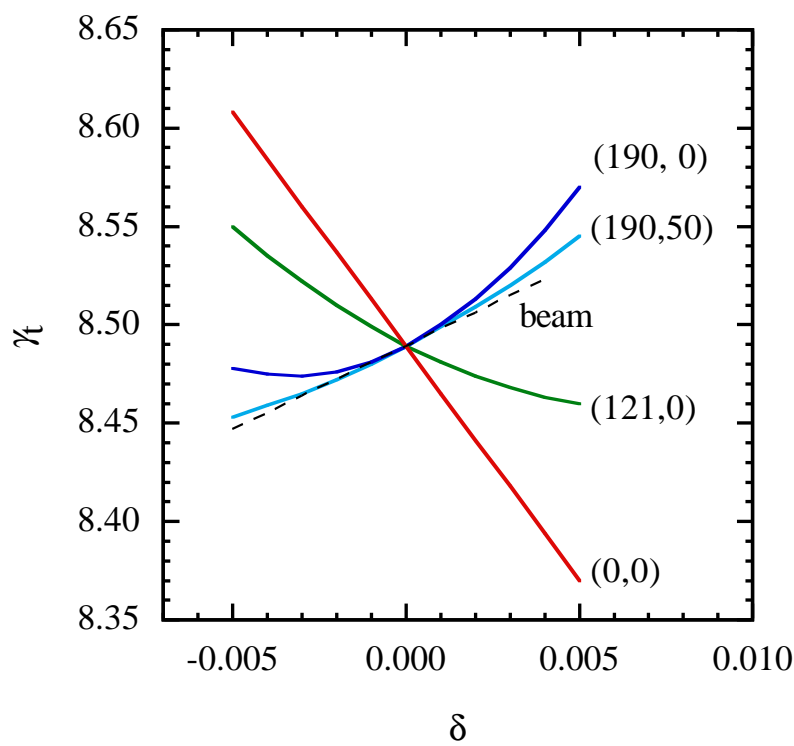

FIG. 1. (Color) Predicted momentum dependence of $\gamma_{t}$ as a function of horizontal sextupole and octupole current $\left(I_{\mathrm{Hs}}, I_{\mathrm{O}}\right)$. of $\gamma_{t}$ may be somewhat inaccurate due to the large orbit distortions from the $\gamma_{t}$ jump.

The longitudinal space charge voltage $V_{\text {sc }}$ seen by a beam particle is proportional to the slope of the current density $d I_{p} / d z$. It becomes important if the bunching is done slowly, in $n$ turns, since the distortion of the bunch is due to $V_{\mathrm{sc}} n$; however, it seems possible to reduce these problems if the final bunching is done in a few turns [13]. Since space charge effects depend on the derivative of a roughly symmetric charge distribution, they result in a shear of the bunch in the $\delta$ direction, which has a linear or cubic dependence on $\phi$. The effects of transition and space charge are shown in Fig. 2, where (a) shows the effects of nonlinear phase shear $\eta \delta$ and (b) shows the effect of space charge, which tends to modulate the energy of the bunch with a term proportional to $d I_{p} / d z$. These simulations were done by tracking the longitudinal motion of particles in the machine using the code ESME [14].

In this experiment, bringing the $\gamma_{t}$ down to the beam $\gamma$ lowers the synchrotron frequency, making the bucket much higher. As the suddenly mismatched bunch rotates in the taller bucket, it becomes taller and more narrow in phase $\phi$ or time. As one moves closer to transition one would expect to find a more narrow final bucket; however, the synchrotron frequency can become so low that bunching would not occur at all.

\section{OPERATION OF THE AGS}

The operating mode of the AGS is shown in Fig. 3. The maximum beam energy was reduced from the normal $28 \mathrm{GeV}$ to around $7 \mathrm{GeV}$ by shortening the acceleration period, and the $\gamma_{t}$ jump system [14] was modified to give a short flattop period before the transition energy dropped. The beam was flattopped for $300 \mathrm{~ms}$ before the magnet guide field was raised slightly and then ramped down. One bunch was injected from the booster at about $0.08 \mathrm{~s}$ into the acceleration cycle, when $d B / d t \sim 0.4 \mathrm{~T} / \mathrm{s}$, and the beam was in the machine for about $500 \mathrm{~ms}$ total. The rf cavities were operated at $340 \mathrm{kV} /$ turn while there was beam. The bunch current was $3-5 \times 10^{12}$ in a single bunch, and the bunch area was measured to be

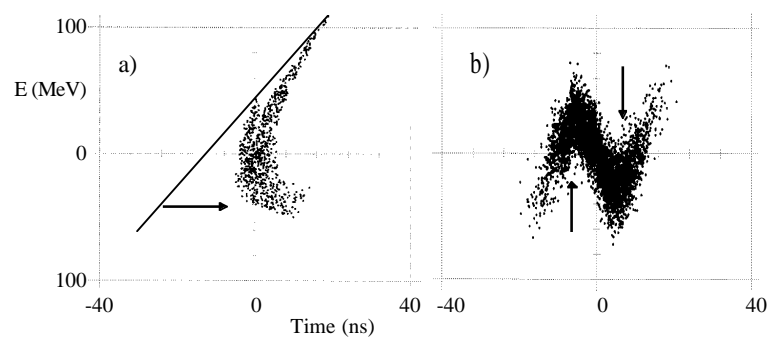

FIG. 2. Effects of nonlinear $\eta$ and space charge on the final phase space distributions. (a) Shows the effect of a quadratic horizontal shear which occurs when $\alpha_{1} \neq-1.5$, and (b) shows the effect of vertical shear from strong space charge effects. 


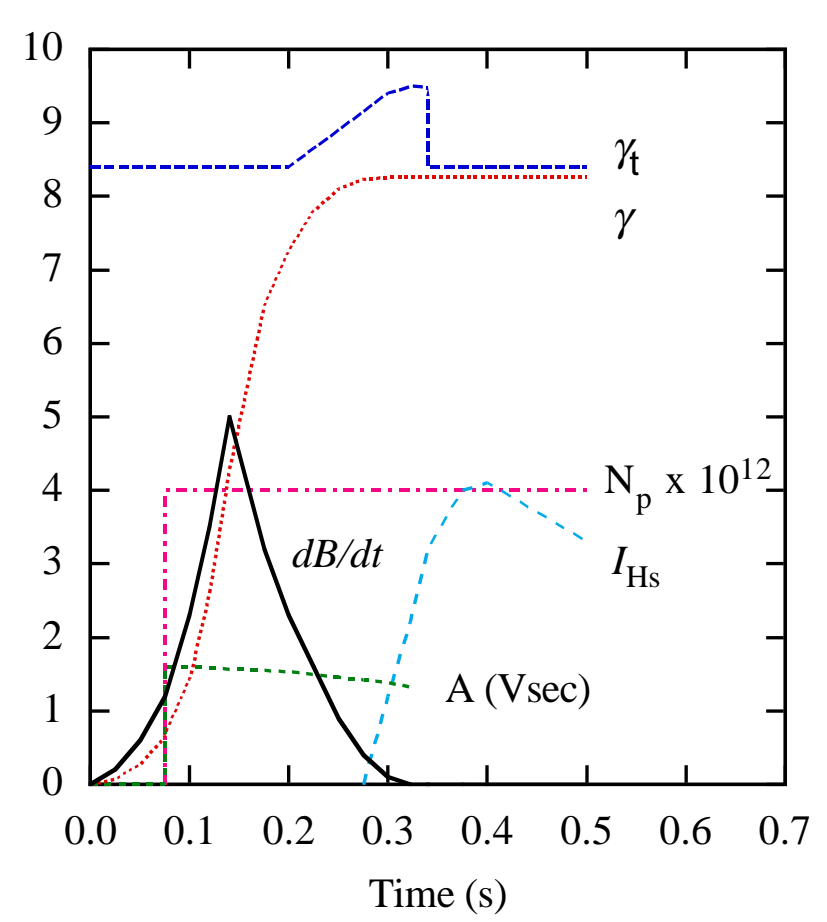

FIG. 3. (Color) The operating mode of the AGS, showing the magnet ramp $d B / d t$, beam gamma $\gamma$, transition gamma $\gamma_{t}$, sextupole currents $I_{\mathrm{Hs}}$, and longitudinal bunch area A.

$1.5 \pm 0.05 \mathrm{eVs}$, declining during the cycle. Measurements of the bunch area were made by reconstructing the bunch shape by observing the development of the time profile during synchrotron rotations: The data are shown as a function of time from injection in Fig. 4.

Previous work had shown that the $\gamma_{t}$ jump system caused significant changes in the nonlinear momentum compaction factor $\alpha_{1}$, the measured maximum dispersion, and the momentum aperture. Although three sextupole families are present in the ring, the locations of these sextupoles are such that these lattice changes are difficult to modify both before and after the transition energy is moved. In order to evaluate the magnitude of these effects, we remeasured the transition energy as a function

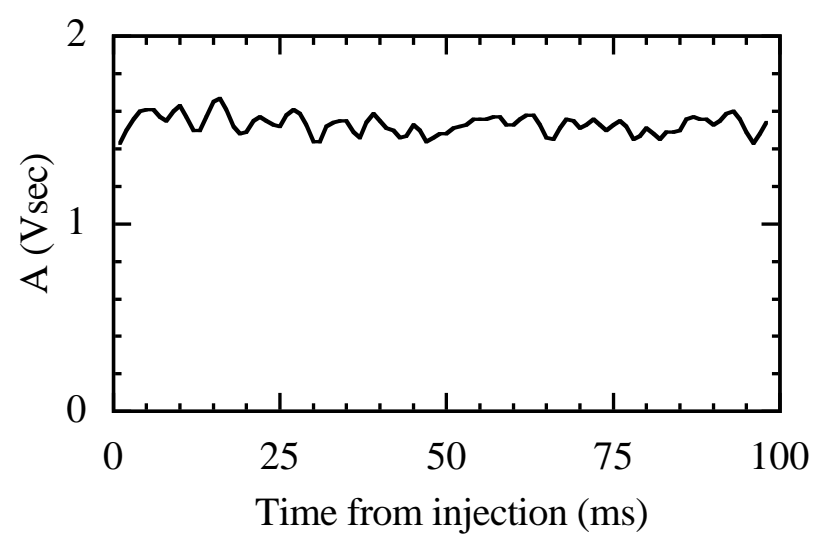

FIG. 4. Bunch area as a function of time from injection. of radial position by measuring beam loss as a function of phase-switch delay time and horizontal sextupole current.

\section{EXPERIMENTAL RESULTS}

The transition energy was first measured relative to the Gauss clock, which gives the instantaneous field in the dipoles as a function of time, for four values of radial displacement. The published value of $\alpha_{1}$ was checked by measuring the radial dependence of beam losses as a function of the time at which the phase of the rf accelerating field was flipped, to compensate for the reversed direction of shear.

The measurements were made by accelerating the beam through transition with the transition jump off and minimizing beam loss by varying the time the rf phase is switched. These data were taken as a function of the horizontal position of the beam and also the horizontal sextupole current. During these measurements, a low emittance beam was used, and the radial position of the beam scanned over the range $\pm 4.6 \mathrm{~mm}$. The measurements were repeated at sextupole currents of 0 and $100 \mathrm{~A}$. The results of these measurements are shown in Figs. 5(a) and 5(b).

The transition energy is obtained from the average of values with the sextupoles on, and is equal to $\gamma_{t}=$ $8.34 \pm 0.05$. The corresponding measurements of $\alpha_{1}$, obtained using Eq. (5), give the results $\alpha_{1}=7.2 \pm 1.5$ with $I_{\mathrm{Hs}}=0 \mathrm{~A}$ and $\alpha_{1}=3.5 \pm 1.5$ with $I_{\mathrm{Hs}}=100 \mathrm{~A}$. These measurements agree roughly with earlier published data [15]; however, time constraints did not permit good statistics. The parameters of the operating machine are also likely to slowly evolve over time as it is tuned for a variety of experiments. There may also be a systematic error in the measurement of $\gamma_{t}$ by this method due to the optimum time delay between the phase flip and transition time, because of the nonlinear and asymmetric behavior of the bunches near transition.

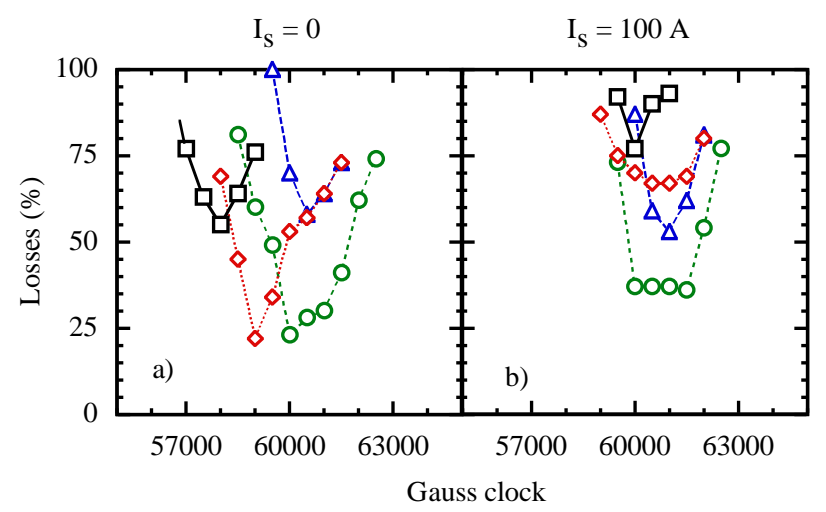

FIG. 5. (Color) Losses going through transition as a function of the time at which the phase of the rf is flipped, and machine field $B$. Here squares, diamonds, circles and triangles refer to $\Delta r=4.6,1.5,-1.5,-4.6 \mathrm{~mm}$, respectively. (a) $I_{\mathrm{Hs}}=0$, (b) $I_{\mathrm{Hs}}=100 \mathrm{~A}$. 
When the operating mode described in Fig. 3 was implemented, the beam was found to be quite stable near transition. After the first quadrupole synchrotron oscillation, all structure tended to damp out and the beam circulated stably without significant losses or beam blowup. Changes in the beam energy, to move below or above transition, or sextupole settings seemed to make little difference, and the overall structure of the beam was almost unaffected by accelerator parameters. Losses were not accurately recorded, but seemed small $(\sim 5 \%)$, and no particular effort was made to minimize them.

Our data, an example of which is shown in Fig. 6, consist primarily of peak beam current and instantaneous current plotted against machine phase (mountain range) plots from $10 \mathrm{~ms}$ before the end of the $\gamma_{t}$ jump to $40 \mathrm{~ms}$ after, using the wall current monitor [17]. The data were read into a LeCroy 9350 digital oscilloscope controlled by LABVIEW, and the data was digitized at intervals of $1 \mathrm{~ns}$. Measurements were made over a range of $0.97<\gamma / \gamma_{t}<1.02$. Some data were taken showing debunching at the end of the run. The bandwidth of the system was determined by cable attenuation.

Measurements of the bunch shape as a function of time were used to measure the synchrotron period and synchrotron frequency, which was then used to estimate $\gamma_{t}$. The result of these measurements was $\gamma_{t}=8.43 \pm$ 0.05 , with the error calculated from the scatter of the individual measurements. A typical set of data used to determine the synchrotron period is shown in Fig. 7.

The results of the bunching tests are shown in Fig. 8, where a typical bunch shape is plotted both before and after the $\gamma_{t}$ was suddenly changed. The narrow final shape is noticeably asymmetric because it was impossible to entirely tune out the chromatic effects mentioned above. Runs further from transition showed a number of synchrotron oscillations, and the bunch

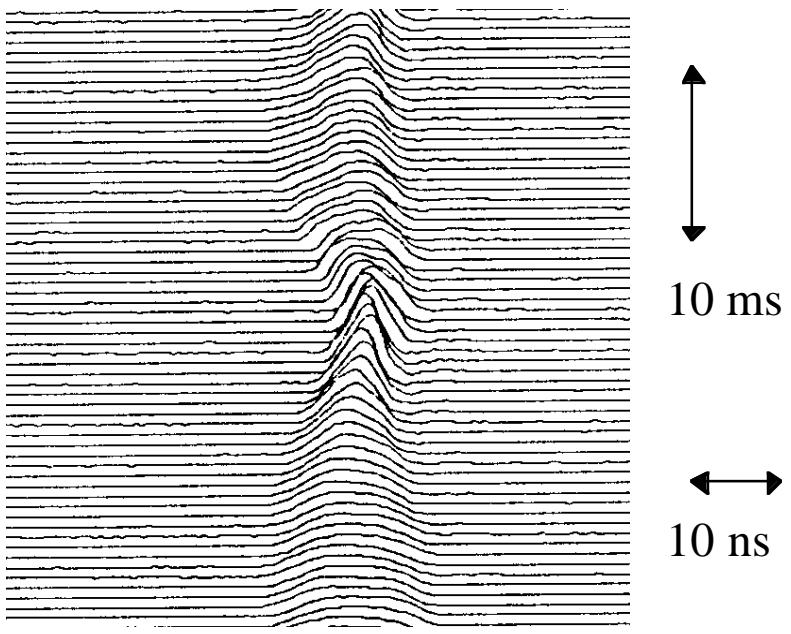

FIG. 6. Mountain range plot of the bunch after the transition energy was dropped to the beam energy. length in later minima was always longer than the first minimum. Closer to transition the bunch length contracted to a minimum and then expanded to roughly the initial value. There seemed to be little emittance blowup. Losses were a function of sextupole currents, but there was insufficient time to explore the dependence in detail. Although the minimum bunch width decreased with increasing sextupole current, it was found that the sextupoles required only about half of the current predicted by MAD to optimize $\eta(\delta)$, and we were not able to produce a value of $\alpha_{1}=-1.5$, which is expected to produce the minimum bunch length.

The initial bunch shape could be approximated either with a Gaussian with an rms width of $\sigma \sim 9$ ns or a twodimensional polynomial of the form

$$
\rho(\delta, \phi) \propto 1-\left[\left(\phi / \phi_{\max }\right)^{2}+\left(\delta / \delta_{\max }\right)^{2}\right]^{(1.75 \pm 0.25)},
$$

which is projected onto the time axis to give a slightly more peaked profile. The shape after bunching tended to be somewhat asymmetric, as would be expected if $\alpha_{1}$ was not optimized. There were no dipole oscillations and phase mismatch seemed negligible.

The final bunch width was, of course, a function of the bunch dimensions before the $\gamma_{t}$ jump was turned off. In one run, the initial bunch length was increased producing smaller initial momentum spread. This was done by pumping the longitudinal coherent quadrupole mode by modulating the amplitude of the rf voltage at twice the synchrotron frequency. The modulation was a $320 \mathrm{~Hz}$ square wave of $20 \%$ amplitude synchronized so the bunch was longest when the $\gamma_{t}$ jump was fired. When the $\gamma_{t}$ jump was then turned off, the bunch rotated in the

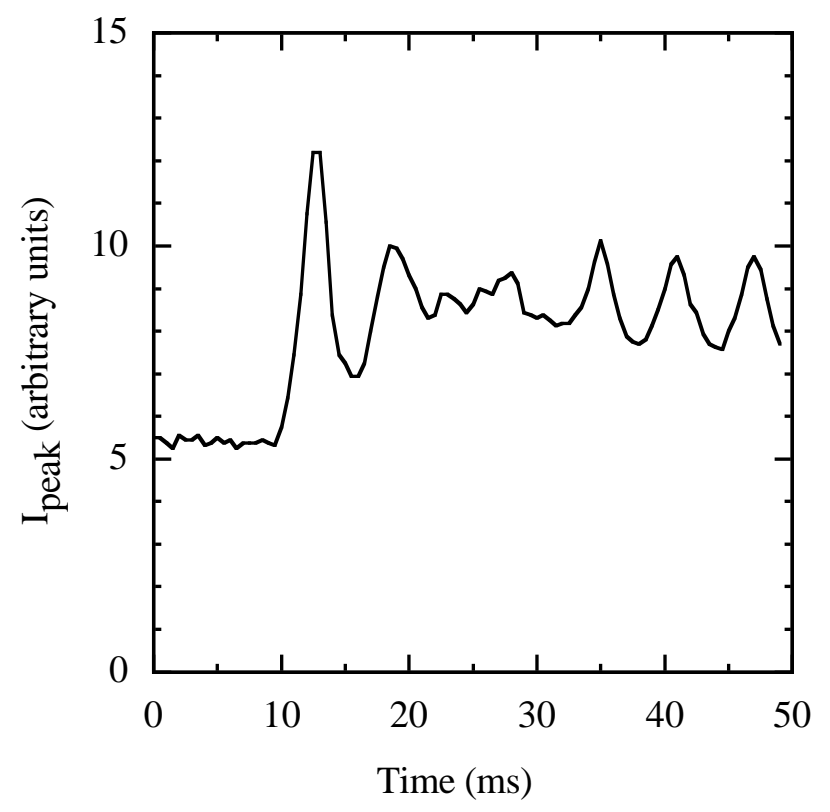

FIG. 7. Peak current as a function of time in the acceleration cycle. Bunching starts at $8 \mathrm{~ms}$. 


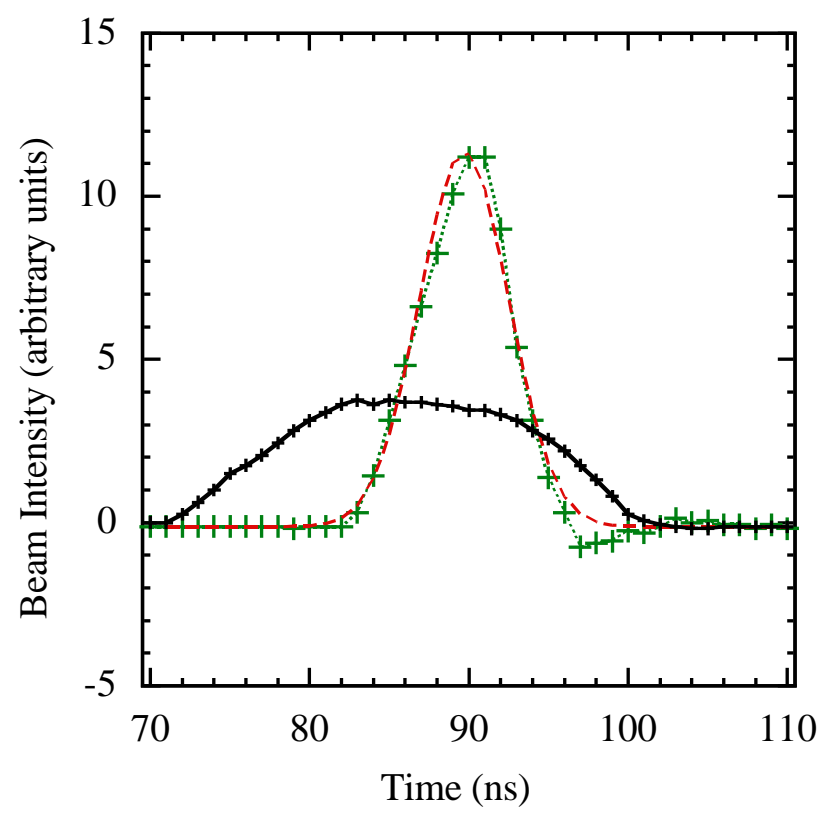

FIG. 8. (Color) Bunch shapes before and after bunching (crosses) showing a final corrected bunch length of $\sigma=2.66 \mathrm{~ns}$ for a $1.5 \mathrm{Vs}$ bunch together with the fitted Gaussian curve (dashed line).

normal way, but the uncorrected width was $\sigma=2.2 \mathrm{~ns}$, about $15 \%$ shorter than bunches obtained without the rf modulation. (The fitted bunch width depended on how much of the wings of the bunch were fitted.) The trace from the digital oscilloscope for this bunch is shown as Fig. 9; we anticipate that optimization would produce even shorter bunches.

The fitted shape shown in Fig. 8 contains corrections for attenuation loss in the cables. This attenuation can be detected in a number of ways, including a small $(\sim 5 \%)$ bunch width dependence of the total bunch area, which occurs primarily in higher frequency components. This

\section{$\mathrm{I}_{\mathrm{B}}$ (Arbitrary units)}

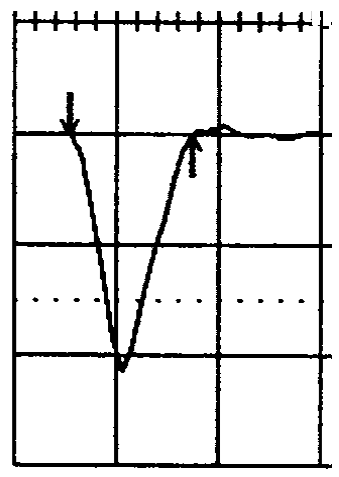

\section{Time (10ns/div)}

FIG. 9. Digital oscilloscope trace of final pulse shape using pulsed rf and the $\gamma_{t}$ jump, giving $\sigma \sim 2.2 \mathrm{~ns}$ (uncorrected) and $11.95 \mathrm{~ns}$ full width (between arrows). correction was made by Fourier analyzing the measured bunch shape, correcting the individual Fourier coefficients for attenuation loss, then summing the series to produce a corrected final bunch shape. The corrections were also obtained by measuring attenuation and dispersion of the cable from the wall current monitor to the control room of the AGS. A 300 ps signal was transmitted through the apparatus and the resulting shape was fitted. The result of this test showed that 0.2 ns of the fitted width shown in all measurements is due to cable dispersion and attenuation.

In order to examine $\eta \delta$ more directly, we also measured debunching when $V_{\mathrm{RF}}$ was suddenly reduced to zero. This was done at two energies to be sensitive to $\eta(\gamma)$. The data, shown in Fig. 10, have sharp discontinuities at the ends of the bunch which make the determination of the boundaries fairly unambiguous. An asymmetry of the bunch shape as it lengthens is consistent with $\alpha_{1} \neq-1.5$. Simulations described below can be used to determine values for $\eta$ and $\gamma_{t}$ from estimates of the full momentum spread of the beam. If the full width of the bunch is assumed to be $\delta=0.025$, obtained from simulations below, debunching data give a value of $\gamma_{t}=8.45 \pm 0.04$, which is consistent with other methods, but more indirect.

We assume that the beam induced voltage $V=I_{b} Z$, where $I_{b}<100 \mathrm{~A}$ is the beam current and the impedance of the vacuum chamber $Z \sim 100 \Omega$ is small compared with the voltage required to contain the bunch in a bucket. As the bunch spreads, the instantaneous current and the induced voltage become very small and this assumption becomes more exact.

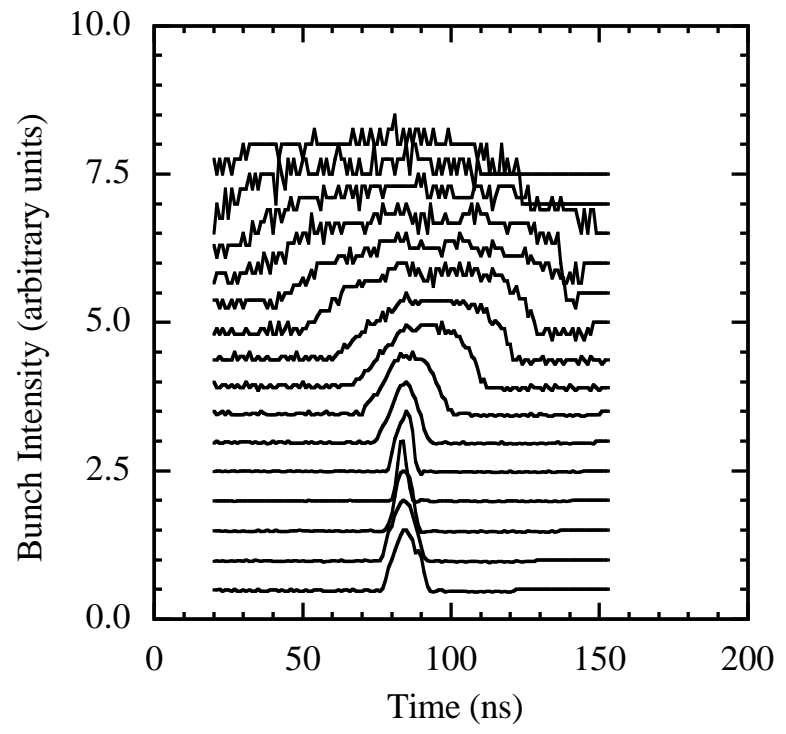

FIG. 10. Debunching of the beam when $V_{\text {rf }}$ is set to 0 after the beam is bunched. The vertical scale is arbitrary and the time between traces is $2.5 \mathrm{~ms}$. The traces have been normalized to a constant amplitude. 


\section{DISCUSSION}

In order to understand the mechanism of bunching, we have done simulations using ESME [14]. These calculations track the longitudinal motion of particles in the bunch over the acceleration and bunching cycle with various parameters. The primary variables are $\Delta \gamma=$ $\gamma_{t}-\gamma$ and $\alpha_{1}$. Simulations show that bunching can occur over a period of $1 \mathrm{~ms}$ and produce bunches with large momentum spread (full widths of $\delta=0.024$ ) and narrow widths. An example of these simulations is shown in Fig. 11, which approximates the parameters shown in Fig. 8. These simulations fairly closely match the structure of the data; however, the accuracy of the fit seems to be a function of a number of variables such as the parametrization of the density of charge within a bunch. The shape of the shortest bunch that can be produced is sensitive to $\alpha_{1}$ and can be used to estimate this parameter; however, the bunch shapes seen do not precisely match those produced in simulations, perhaps indicating higher order terms $\left(\eta \propto \delta^{2}\right)$. The data are consistent with $\alpha_{1}=0.0 \pm 1.0$. The total height of the bunch $\delta \sim 0.024$ can circulate in the AGS, which has a total momentum acceptance of $\delta \sim \pm 0.025$, assuming no closed orbit distortion. Since the momentum acceptance is sufficiently large, we expect that a further reduction in the bunch length is possible; however, this might be accompanied with increased losses, if the beams were large.

The measurements of $\gamma_{t}$ and $\alpha_{1}$ by a variety of methods are generally consistent with previous measurements, which give $\gamma_{t}=8.45, \alpha_{1}=2.5$ from Wei et al. [16], and $\gamma_{t}=8.49$ from MAD. It should be noted that all

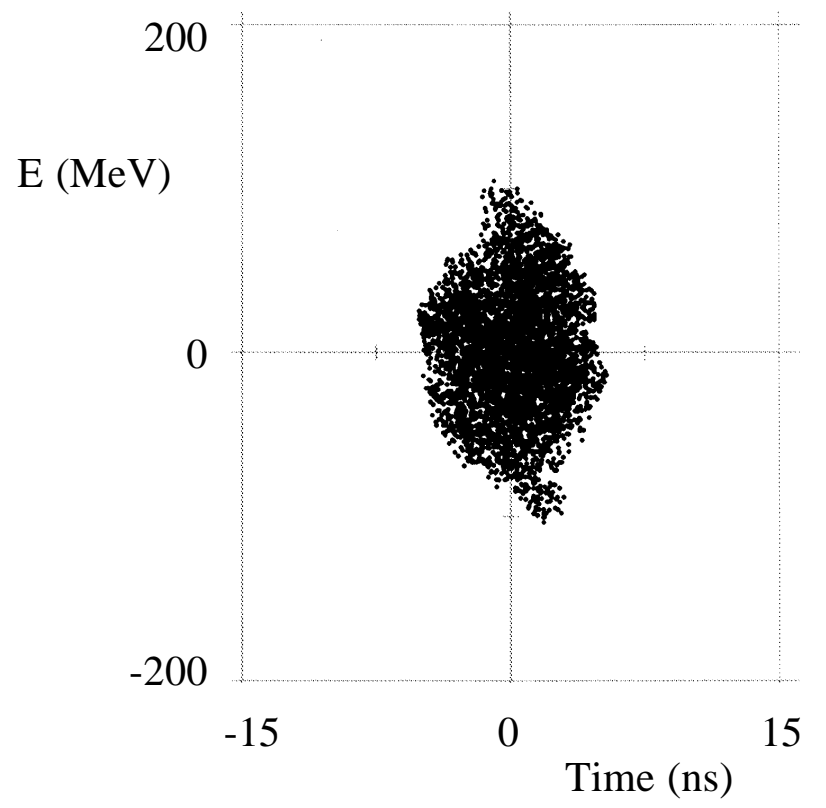

FIG. 11. The final phase space after $\delta_{t}$ is moved close to the beam energy $(\gamma=8.40)$. the measurements are somewhat indirect and the real machine parameters may vary somewhat from the models. We assume that the most accurate method of measuring $\gamma_{t}$ is by means of the synchrotron frequency, since systematic errors should be small and the measurements are comparatively straightforward.

A proton bunch width of $\sigma \sim 2.0-2.7$ ns begins to be short enough to consider the use of this method for the proton driver of the muon collider. The measurements here imply that $\alpha_{1}$ is perhaps somewhat larger than previously measured and more sextupole and octupole currents would permit smaller values of $\eta(\delta)$ which would, in turn, produce shorter bunches. Nevertheless, these data show the shortest high current bunches measured in the AGS.

While space charge effects are much smaller here than they would be for the proton driver, it is assumed that the use of FMC lattices would allow very fast motion of the transition energy, which could make bunching fast enough so that this effect might not significantly perturb bunching, even for much higher currents. One could also jump above transition, where space charge would help bunching, or insert inductance into the ring to compensate the effect.

While the measurements made here apply directly to the design of a proton driver for a muon collider, the stability of high current bunches close to transition is also relevant to the design of isochronous rings for other applications.

\section{CONCLUSIONS}

During a short run, we were able to explore the operation of the AGS when it was flattopped close to transition. It was found that the bunch length could be reduced by a factor of $\sim 3$ by using the $\gamma_{t}$ jump system to suddenly drop the transition energy to the beam energy, and the beam seemed stable over the $40 \mathrm{~ms}$ we observed it. We also measured $\gamma_{t}$ using (i) the time of phase flip, (ii) the synchrotron frequency, and (iii) debunching time, producing rough agreement with previous measurements and with MAD calculations for the lattice. Measurements of $\alpha_{1}$ generally agreed with earlier data, but the precision of the data was limited by statistics and measurement time. Attempts to tune $\alpha_{1}$ with sextupoles and octupoles were less successful due to power supply limitations. The stability of the beam circulating near transition was unexpected, and argues for the stability of high current isochronous storage rings.

We were unable to systematically explore possible methods of bunch manipulation due to time constraints; nevertheless, the $\sigma \sim 2.0 \mathrm{~ns}$ bunch length produced by this method is sufficiently short to be useful for the proton driver of the muon collider. Modification of the bunch shape before rotation, for example, seems useful, but was not optimized. Effects of space charge were also not 
studied systematically, and measurements with significant space charge effects would be highly desirable, as would the ability to move the transition energy in two directions, which could be used to produce rapid bunching.

Designs for the proton driver include plans for 4-5 MHz rf systems which would have bunches half the length of AGS bunches. In addition, the final energy in these designs is at least twice that of the AGS transition energy. Both of these factors should make bunching somewhat easier in the new machine.

\section{ACKNOWLEDGMENTS}

We thank the operating crew of the AGS for their cooperation in performing these measurements. We also acknowledge useful discussions with C. Johnstone, D. Neuffer of Fermilab, M. Yoshi and Y. Mori of KEK, and B. Autin of CERN. This work was supported by the U.S. Department of Energy.

[1] D. Neuffer, Part. Accel. 14, 75 (1983).

[2] The $\mu^{+} \mu^{-}$Collider Collaboration, in Proceedings of the Snowmass Workshop, Snowmass '96 (SLAC, Menlo Park, CA, 1997).

[3] D. Cline, B. Norum, and R. Rossmanith, in Proceedings of the European Particle Accelerator Conference, Sitges, Spain, 1996 (Institute of Physics Publishing, Philadelphia, PA, 1996), p. 867.

[4] M. Brennan, in Proceedings of the Workshop on the Proton Driver for the Muon Collider, Argonne, 1996 (Argonne National Laboratory, Argonne, IL, 1996).

[5] J. Griffin, Fermilab (private communication).

[6] The Proton Source Summer Study Group, Fermilab Technical Note, Fermilab-TM-2021, edited by S.D. Holmes (1997).
[7] F. Mills, Fermilab (private communication).

[8] R. Cappi, J. P. Delahaye, K. H. Reich, IEEE Trans. Nucl. Sci. 28, 2389 (1981).

[9] S. Y. Lee, K-Y. Ng, and D. Trbojevic, Phys. Rev. E 48, 3040 (1993).

[10] D. Trbojevic, M. Brennan, E. O. Courant, T. Roser, S. Peggs, C. Johnstone, K-Y. Ng, J. Norem, and M. Popovic, in Proceedings of the 17th IEEE Particle Accelerator Conference, Vancouver, Canada, 1997 (to be published).

[11] J. Wei, Ph.D. thesis, State University of New York at Stony Brook, 1994.

[12] H. Grote and F.C. Iselin, The MAD program (methodical accelerator design), Version 8.10, User's Reference Manual, CERN SL 90-13 AP Rev 3 (1993).

[13] J. Norem, C. Ankenbrandt, J. Griffin, C. Johnstone, S. Y. Lee, K-Y. Ng, M. Popovic, M. Brennan, T. Roser, J. Wei, and D. Trbojevic, in Proceedings of the 17th IEEE Particle Accelerator Conference, Vancouver, Canada, 1997 (to be published).

[14] J. MacLachlan and J.F. Ostiguy, in Proceedings of the 17th IEEE Particle Accelerator Conference, Vancouver, Canada, 1997 (to be published).

[15] W. K. Van Asselt, L.A. Ahrens, J.M. Brennan, A. Dunber, E. Keith-Monnia, J. T. Morris, and M. J. Syphers, Proceedings of the 1995 Particle Accelerator Conference, Dallas (IEEE, Piscataway, NJ, 1996), p. 3022.

[16] J. Wei, M. Brennan, L. A. Ahrens, M. M. Blaskiewicz, D-P. Deng, W. W. MacKay, S. Peggs, T. Satogata, D. Trbojevic, A. Werner, and W.K. van Asselt, in Proceedings of the 1995 Particle Accelerator Conference, Dallas (IEEE, Piscataway, NJ, 1996), p. 3334.

[17] W. K. van Asselt and L. A. Ahrens, in Beam Instrumentation Workshop, edited by George H. Mackenzie, Bill Rawnsley, and Jana Thomson, AIP Conf. Proc. No. 333 (AIP, New York, 1995), p. 473. 